\title{
Presurgical Brain Mapping of the Ventral Somatomotor Network in Patients with Brain Tumors Using Resting-State fMRI
}

(D) N. Yahyavi-Firouz-Abadi, (D).J. Pillai, (D).A. Lindquist, (DV.D. Calhoun, (DS. Agarwal, DR.D. Airan, (D) B. Caffo, (D) S.K. Gujar, and

(1) H.I. Sair

\section{ABSTRACT}

BACKGROUND AND PURPOSE: Resting-state fMRI readily identifies the dorsal but less consistently the ventral somatomotor network. Our aim was to assess the relative utility of resting-state fMRI in the identification of the ventral somatomotor network via comparison with task-based fMRI in patients with brain tumor.

MATERIALS AND METHODS: We identified 26 surgically naïve patients referred for presurgical fMRI brain mapping who had undergone both satisfactory ventral motor activation tasks and resting-state fMRI. Following standard preprocessing for task-based fMRI and resting-state fMRI, general linear model analysis of the ventral motor tasks and independent component analysis of resting-state fMRI were performed with the number of components set to 20,30,40, and 50. Visual overlap of task-based fMRI and resting-state fMRI at different component levels was assessed and categorized as full match, partial match, or no match. Rest-versus-task-fMRI concordance was calculated with Dice coefficients across varying fMRI thresholds before and after noise removal. Multithresholded Dice coefficient volume under the surface was calculated.

RESULTS: The ventral somatomotor network was identified in $81 \%$ of patients. At the subject level, better matches between resting-state fMRI and task-based fMRI were seen with an increasing order of components (53\% of cases for 20 components versus $73 \%$ for 50 components). Noise-removed group-mean volume under the surface improved as component numbers increased from 20 to 50 , though ANOVA demonstrated no statistically significant difference among the 4 groups.

CONCLUSIONS: In most patients, the ventral somatomotor network can be identified with an increase in the probability of a better match at a higher component number. There is variable concordance of the ventral somatomotor network at the single-subject level between resting-state and task-based fMRI.

ABBREVIATIONS: BOLD = blood oxygen level-dependent; ICA = independent component analysis; rs-fMRI = resting-state fMRI; tb-fMRI = task-based fMRI; VSMN = ventral somatomotor network; VUS = volume under the surface

$\mathbf{F}_{\text {gie }}^{\text {un }}$ unctional MRI is widely used as a noninvasive tool for presurgical localization of the eloquent cortex, typically involving somatomotor and language mapping. Mapping of these eloquent brain areas with fMRI correlates well with invasive methods such

Received June 5, 2016; accepted after revision December 25.

From the Department of Radiology (N.Y.-F.-A.), Mid-Atlantic Permanente Medical Group of Kaiser Permanente, Kensington, Maryland; Division of Neuroradiology, (N.Y.-F.-A., J.J.P., S.A., R.D.A., S.K.G., H.I.S.), The Russell H. Morgan Department of Radiology and Radiological Sciences, Johns Hopkins University School of Medicine Baltimore, Maryland; Department of Biostatistics (M.A.L., B.C.), Johns Hopkins University, Baltimore, Maryland; and The Mind Research Network (S.A., V.D.C.), Departments of Electrical and Computer Engineering, University of New Mexico, Albuquerque, New Mexico.

This work was supported in part by the Radiological Society of North America Research and Education Foundation-Carestream Health/RSNA Research Scholar Grant to H.I.S. (grant No. RSCH1420).

Previously presented in part at: Annual Meeting of the American Society of Neuroradiology and the Foundation of the ASNR Symposium, April 25-30, 2015; Chicago, Illinois. as intraoperative electrocortical stimulation ${ }^{1}$ and can result in reduced surgical time, increased extent of resection, and decreased craniotomy size. ${ }^{2}$ In addition, postoperative morbidity correlates with the distance of the resection margin from fMRIidentified eloquent cortex. ${ }^{3}$ In current clinical practice, changes in blood oxygen level-dependent (BOLD) signal are measured across time as the patient performs a specific task (ie, task-based fMRI [tb-fMRI]). ${ }^{4}$ These BOLD signal changes reflect characteristic hemodynamic responses to neural activity. However, tbfMRI has several limitations. Primarily, accurate localization of function is dependent on the patient's cooperation and ability to

Please address correspondence to Noushin Yahyavi-Firouz-Abadi, MD, Division of Neuroradiology, The Russell H. Morgan Department of Radiology and Radiological Sciences, Johns Hopkins University School of Medicine, 600 North Wolfe St, Phipps B-112, Baltimore, MD 21287; e-mail: nyahyavi@gmail.com; @nyahyavi

三 Indicates article with supplemental on-line table.

http://dx.doi.org/10.3174/ajnr.A5132 
adequately perform the task, which can be an important limitation in those with physical or mental debilitation or those who cannot understand the instructions such as children. The patient must be awake and cooperative during the task; therefore, sedation cannot be used; this feature is a limitation in the pediatric population. Furthermore, due to the inherently low signal-tonoise ratio of this technique, long and repeated acquisitions are often required to ensure adequate data sampling for analysis. ${ }^{5,6}$

Resting state fMRI (rs-fMRI) is a promising method of assessing brain function that can overcome some of the limitations of tb-fMRI. In rs-fMRI, spontaneous fluctuations in BOLD signal are measured across time while no specific task is performed. Temporal correlations of these spontaneous fluctuations can be organized into spatially distinct intrinsic networks. ${ }^{5}$ These networks are now described as resting-state networks, many of which have topography similar to that of networks engaged in sensory, motor, and cognitive tasks. ${ }^{7}$ These intrinsic resting-state networks persist, though somewhat modified, in states of decreased awareness such as sleep ${ }^{8}$ or sedation. ${ }^{9}$ Rs-fMRI has thus gained interest as a potential viable alternative to tb-fMRI, especially in pediatric or cognitively/neurologically impaired patients. Multiple studies have investigated the potential role of rs-fMRI in presurgical mapping of somatomotor ${ }^{5,10-12}$ and language $e^{2,6,13}$ networks. In a limited number of subjects, moderate overlap between rs-fMRI and tb-fMRI was found for mapping of the motor cortex. ${ }^{5,11,12}$ Furthermore, there is good qualitative concordance between intraoperative cortical stimulation and rs-fMRI in the localization of the eloquent motor cortex. ${ }^{10}$ With quantitative analysis, rs-fMRI and tb-fMRI perform comparably, but the shortest distance to stimulation points is observed for tb-fMRI. ${ }^{11}$ Rs-fMRI has been shown to identify a larger pattern of the motor network compared with tb-fMRI. ${ }^{11,14}$ In addition, localization by rs-fMRI and tb-fMRI may include different parts of the sensorimotor network. ${ }^{11,15}$ While the motor system at large has been the target of most prior investigations, distinct subnetworks of the motor network exist. We narrowed our focus to the ventral motor area, which largely reflects oral somatomotor function. Three recent studies evaluated the concordance of rs-fMRI and tb-fMRI in the localization of the face representation area of the primary motor cortex in addition to limb motor areas in a limited number of patients with a variety of brain lesions in different locations. ${ }^{11,12,16}$

To our knowledge, no study has specifically investigated the concordance between tb-fMRI activation and the rs-fMRIderived ventral somatomotor network (VSMN) maps in a large cohort of patients with lesions close to or involving the VSMN. We hypothesized that the ventral motor network can be identified in patients with brain tumors and that there is good concordance with tb-fMRI with a tongue motor paradigm. In addition, we hypothesized that a higher number of components in independent component analysis (ICA) may yield a better concordance between rs-fMRI and tb-fMRI.

\section{MATERIALS AND METHODS Study Subjects}

The institutional review board approved this retrospective study. Searching the Radiology Information Systems, we identified pa- tients who underwent fMRI for presurgical brain mapping between January 1, 2009, and July 31, 2014. Fifty-eight patients underwent rs-fMRI in addition to tb-fMRI during the same imaging session. Seventeen patients had a prior history of a brain operation (including biopsy) and were excluded. One patient had imaging features characteristic of an arteriovenous malformation and was excluded. At the Johns Hopkins Hospital, tb-fMRI for presurgical mapping is tailored for each patient, predominantly based on the location of their tumor and the relation of eloquent regions along the expected surgical trajectory and in the immediate vicinity of the lesion. Among the remaining 40 patients, 26 with brain tumors involving or in close proximity to the ventral somatomotor network who had undergone both tb-fMRI for localization of the VSMN and rs-fMRI were included for final analysis (age range, 21-69 years; mean age, 43.6 years; 15 men and 11 women).

\section{Brain Tumor Characterization}

Tumor location, volume (in cubic millimeters), pathology, and World Health Organization histologic grade (when available) were recorded by a subspecialty board-certified neuroradiologist for each patient. Lesion volume was measured by manually drawing the ROI on FLAIR images in the Medical Image Processing, Analysis, and Visualization application software (MIPAV; National Institutes of Health, Bethesda, Maryland; http://mipav.cit. nih.gov). For high-grade brain tumors, we assessed the entire region of T2 signal abnormality, which represents a combination of infiltrative neoplasm and vasogenic edema.

\section{MR Imaging}

A 3T Tim Trio system (Siemens, Erlangen, Germany) with a 12channel head matrix coil was used. Structural images included a 3D $\mathrm{T} 1$ sequence $(\mathrm{TR}=2300 \mathrm{~ms}, \mathrm{TI}=900 \mathrm{~ms}, \mathrm{TE}=3.5 \mathrm{~ms}$, flip angle $=$ $9^{\circ}$, FOV $=24 \mathrm{~cm}$, acquisition matrix $=256 \times 256 \times 176$, section thickness $=1 \mathrm{~mm})$ and a $2 \mathrm{D}$ T2 FLAIR sequence $(\mathrm{TR}=9310 \mathrm{~ms}$, $\mathrm{TI}=2500 \mathrm{~ms}, \mathrm{TE}=116 \mathrm{~ms}$, flip angle $=141^{\circ}, \mathrm{FOV}=24 \mathrm{~cm}$, acquisition matrix $=320 \times 240 \times 50$, section thickness $=3 \mathrm{~mm}$ ). Functional T2*-weighted BOLD images for both tb-fMRI and rsfMRI were acquired by using 2D gradient-echo echo-planar imaging $\left(\mathrm{TR}=2000 \mathrm{~ms}, \mathrm{TE}=30 \mathrm{~ms}\right.$, flip angle $=90^{\circ}, \mathrm{FOV}=24 \mathrm{~cm}$, acquisition matrix $=64 \times 64 \times 33$, section thickness $=4 \mathrm{~mm}$, section gap $=1 \mathrm{~mm}$, interleaved acquisition). Instructions for rs-fMRI were the following: try to keep still, keep your eyes closed, and do not fall asleep. For rs-fMRI, 180 volumes were acquired (6 minutes).

\section{Tongue Motor Task}

The face representation area of the primary motor cortex was mapped by using the vertical tongue movement task as a robust and spatially extensive representative of the ventral/face motor network. ${ }^{17,18}$ The duration of the task is 3 minutes and consists of 3 cycles of 30 -second blocks of rest alternating with 30-second blocks of repetitive vertical tongue movement. For each patient, a board-certified neuroradiologist with experience in fMRI provided instructions and practice sessions on the tongue motor task outside the scanner. The quality of the tbfMRI maps was monitored in real-time by the neuroradiologist monitoring the session. Per protocol, if real-time maps dem- 


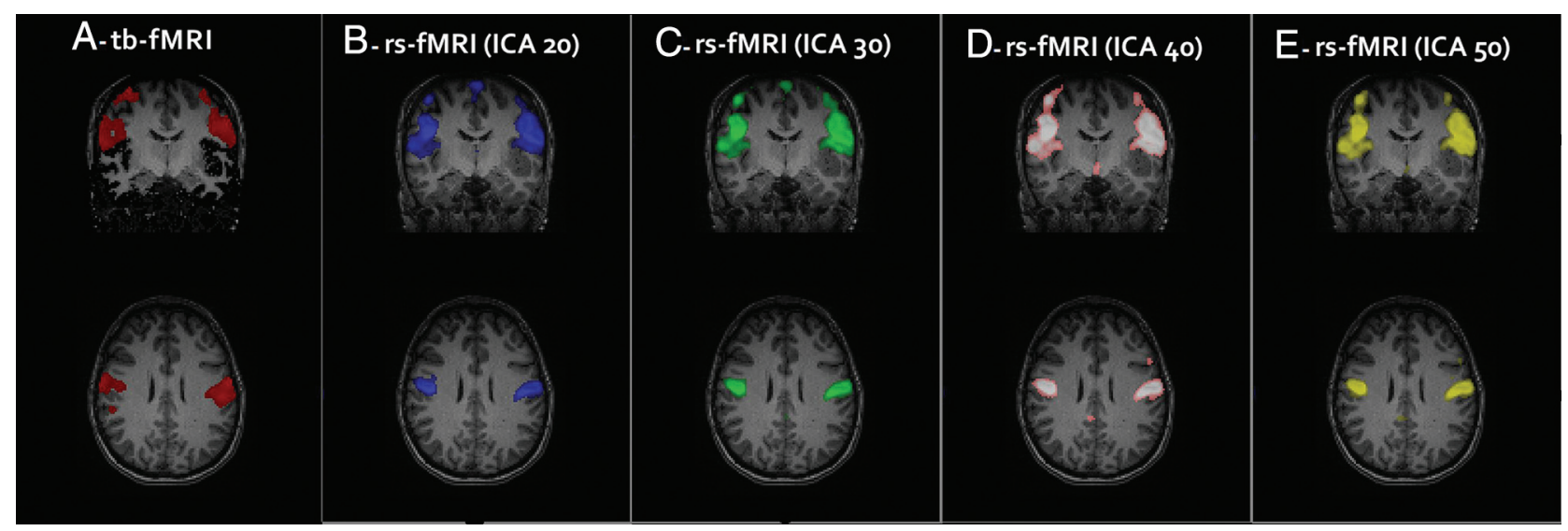

FIG 1. Task-based fMRI map demonstrating activation of the VSMN with the vertical tongue movement task in a single subject (A). Rs-fMRI maps with the best visual correlates to tb-fMRI at different ICA groups $(B-E)$ were selected.

onstrated suboptimal activation or excessive noise, the task was repeated. Tasks were presented with the environment implemented in the Prism Acquire software (Prism Clinical Imaging, Elm Grove, Wisconsin). Only runs that met quantitative quality control criteria $(<2-\mathrm{mm}$ net head displacement along any axis) were considered.

\section{Image Processing}

fMRI data were processed by using Statistical Parametric Mapping, Version 8 (SPM8 software; http://www.fil.ion.ucl.ac.uk/ spm/software/spm12) and custom Matlab (MathWorks, Natick Massachusetts) scripts. Processing of tb-fMRI included sectiontiming correction, motion correction, normalization to a Montreal Neurological Institute 152 template, and spatial smoothing included a 6-mm full width at half maximum Gaussian kernel.

For processing the rs-fMRI data, section-timing correction and motion correction were performed. The motion-correction step included registration of tb-fMRI and rs-fMRI to each other. The ArtRepair toolbox (http://cibsr.stanford.edu/tools/humanbrain-project/artrepair-software.html $)^{19}$ was used to detect volumes with large shifts in global average signal intensity, which include contributions from scan-to-scan motion. The outlier volumes and additional volumes recommended for deweighting in ArtRepair were tagged for subsequent removal from analysis (ie, scrubbing). The rs-fMRI data were then linearly detrended. RsfMRI and T1-weighted images were coregistered and normalized to perform physiologic nuisance regression of rs-fMRI with the component-based noise-correction method. ${ }^{20}$ The same transformation matrix was used for normalization between tb-fMRI and rs-fMRI to ensure that spatial comparison between these 2 was valid for each subject. Bandpass filtering from 0.01 to $0.1 \mathrm{~Hz}$ and smoothing were performed with a 6-mm full width at half maximum Gaussian kernel. At the end, previously tagged images by ArtRepair were scrubbed.

\section{Statistical Analysis}

Tb-fMRI Analysis. We used a general linear model analysis for tb-fMRI implemented in SPM8 with the canonical hemodynamic response function convolved with the boxcar function for each task with standard parameters previously described. ${ }^{6}$ High-pass filtering was performed at the default setting to remove drift. The hemodynamic response function-convolved task vectors were input into a design matrix, and a contrast was created to target the associated parameter comparing the hemodynamic response function-weighted time engaged in the task with rest. The SPM $\mathrm{t}$-contrast maps were generated without clustering because these activation maps were subsequently thresholded across multiple levels for comparison as previously described. ${ }^{6}$ Activation maps were reviewed to ensure that ventral motor activation was present.

Rs-fMRI Analysis. Rs-fMRI was analyzed with the Group ICA of the fMRI Toolbox Software (GIFT; http://mialab.mrn.org/ software/gift/). Independent component analysis was performed separately for each subject by using the InfoMax algorithm with ICASSO (http://research.ics.aalto.fi/ica/icasso/) set at 5 repeats, ${ }^{21}$ including selection of the "best run" to ensure consistent estimates. ICA maps were generated for 20, 30, 40, and 50 components, designated here as ICA20, ICA30, ICA40, and ICA50, respectively. Following scrubbing, 1 subject had only slightly $>50$ volumes left; therefore, the maximum number of components was limited to 50. For each ICA group, the component that best represented the VSMN based on overlap with tb-fMRI was selected visually (Fig 1). Any component whose spatial map was specific and limited to the ventral perirolandic cortices corresponding to the tb-fMRI activation maps was considered a "full match." If the component included additional networks outside these regions, it was considered a "partial match," indicating that the VSMN was present but not separated from other networks. If no VSMN was identified, a "no match" designation was assigned to that ICA group for that subject. A mixed-effects logistic regression analysis was performed to see whether there was a significant increase in the probability of getting a full match as a function of the number of ICA components.

Comparison of tb-fMRI and rs-fMRI. The level of tb-fMRI T-map thresholding may affect the degree of rs-tb fMRI concordance. At low thresholds, there will be artificially high concordance due to the introduction of a higher percentage of voxels, many of which may not represent true activation but rather statistical noise that 
exceeds this threshold. Although there are strategies to determine the level of optimum fMRI thresholding, there is no consensus for reproducible results at a subject level. ${ }^{18}$ To minimize this issue, we used a multithresholding technique to compare concordance across a wide range of thresholds as previously described (Fig 2). ${ }^{6,22}$ We calculated Dice coefficients as a quantitative measure of overlap across each threshold to generate a matrix of rsversus-tb overlap (left map, Fig 3). ${ }^{6}$ Dice coefficients vary between 0 and 1 and give an objective evaluation of similarity or concordance between 2 sets of data. At very low thresholds, high Dice coefficients result from overlap of random noise as can be seen in Fig 2. We used a previously described noise-removal method ${ }^{6}$ briefly described here.

For calculation of noise, an ICA component representing the anterior ventricular signal was selected for each subject. After removing negative values, we normalized image maps in value from 0 to 1 , and we subsequently used multiple thresholds of this normalized map to calculate Dice coefficients between ventricular

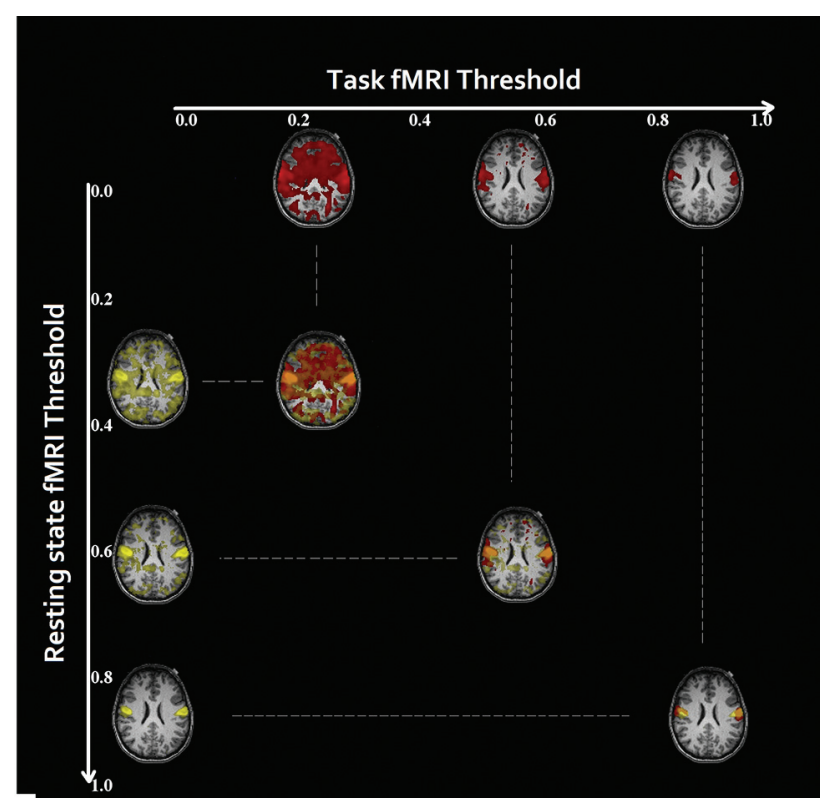

FIG 2. Rs-fMRI ICA maps (yellow) and Tb-fMRI T-maps (red) were thresholded, and 100 threshold maps were generated for each in a single subject. At a low threshold, there is artificially higher overlap (orange) between the maps due to noise. At a very high threshold, the overlap is smaller. "noise" and task maps (middle map, Fig 3). The resultant noiseversus-task Dice map was subtracted from the resting-versus-task Dice map (right map, Fig 3). ${ }^{6}$ Noise-corrected resting stateversus-task-based Dice map volume under the surface (VUS) was calculated across different ICA orders. The VUS measurement collapses this multithresholded dice map into a single variable that can be used as a metric; the VUS is equivalent to the area under the curve in a $2 \mathrm{D}$ graph (such as a receiver operating characteristic curve); however, because the multithresholded dice map has 3 dimensions (the rs-fMRI threshold, the task-threshold, and value), a volume under the surface is computed. One-way ANOVA was performed to determine significant differences in VUS across the 4 ICA orders. Maximum Dice coefficient values at the group level and subject level were calculated.

\section{RESULTS}

\section{Tumors}

The On-line Table summarizes the patients' demographic data, location and volume of lesions, pathology, World Health Organization grade (if applicable), and the distance from the edge of the lesion to the edge of the ventral somatomotor activation cluster based on the clinical task-fMRI maps. Brain lesions were mainly centered in the left cerebral hemisphere (19 patients), and 4 lesions were primarily located in the right cerebral hemisphere. Tumor size ranged from 0.84 to $159.05 \mathrm{~cm}^{3}$ (mean, $41.82 \mathrm{~cm}^{3}$ ).

\section{FMRI Comparison}

Twenty-six patients met the inclusion criteria and were included for analysis. In 21 patients (81\%), rs-fMRI successfully identified a VSMN (Fig 4). Among these, 2 patients were considered a partial match because the VSMN was not distinct from the dorsal somatomotor network. There was an increase in the number of full matches with an increase in the ICA order (14 patients at ICA 20 and 19 patients at ICA 50). In 14 patients, a separate VSMN was identified at all ICA groups. Mixed-effect logistic regression demonstrated a significant increase in the probability of getting a full match as a function of the number of components $(P<.00001)$. This probability is shown in Table 1.

In 5 patients, rs-fMRI failed to identify a VSMN at any of the ICA levels. In 3 of these 5 patients, only the dorsal somatomotor

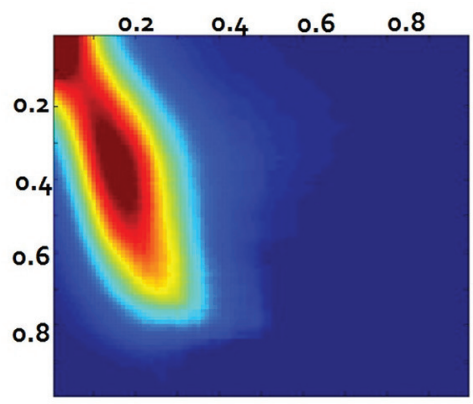

Dice Coefficient matrix

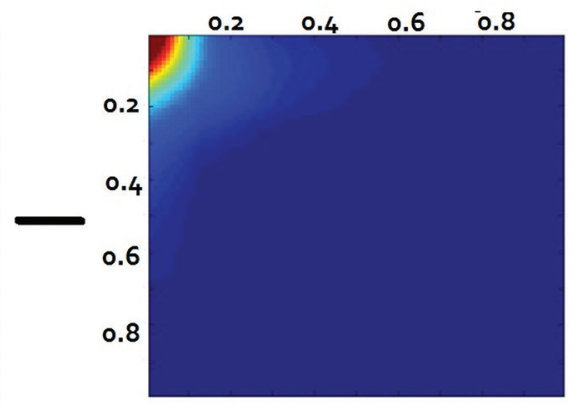

Noise map

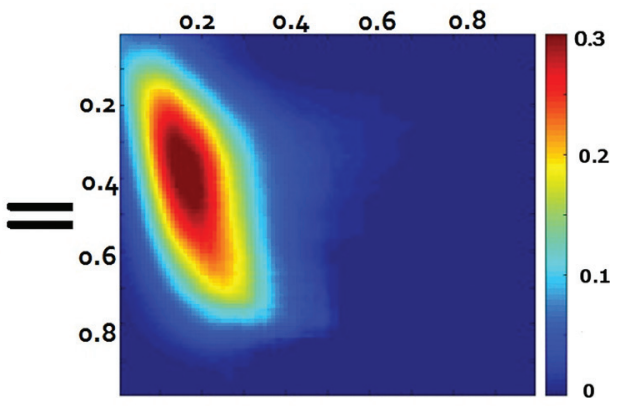

Noise-removed Dice Coefficient map

FIG 3. The Dice coefficient matrix at different thresholds at the subject level between the rs-fMRI (x-axis) at ICA 20 and tb-fMRI (y-axis). An artificially high Dice coefficient is seen in the top left corner of the left map due to overlap of noise. A noise matrix was generated (middle map) and was subtracted (right map). Noise-removed Dice coefficient maps were generated for all the subjects across 4 different ICA orders. 
network was identified. In the remaining 2 patients, no somatomotor network was identified.

Group mean Dice maps for each ICA order are shown in Fig 5. Group mean Dice VUS overall increased with the ICA order (Fig 6); however, 1-way ANOVA demonstrated no significant differences among the 4 ICA groups $(P$ value $=.4)$. The range of Dice coefficient values at the subject level is shown in Table 2. Negative Dice values in these noise-subtracted maps may occur when the concordance between the noise maps and tb-fMRI at low thresholds is greater than the concordance between rs-fMRI and tbfMRI due to the randomness of noise at low thresholds.

\section{DISCUSSION}

A growing number of studies have explored the feasibility of rsfMRI as a substitute or complement to tb-fMRI for presurgical mapping of the eloquent somatomotor cortex in patients with brain tumor. Previous studies have demonstrated the reliability of rs-fMRI for localization of the hand motor area in comparison with tb-fMRI in a limited number of patients, with seedbased $^{10,23}$ or ICA analysis. ${ }^{12}$ Concordance of rs-fMRI with intra-

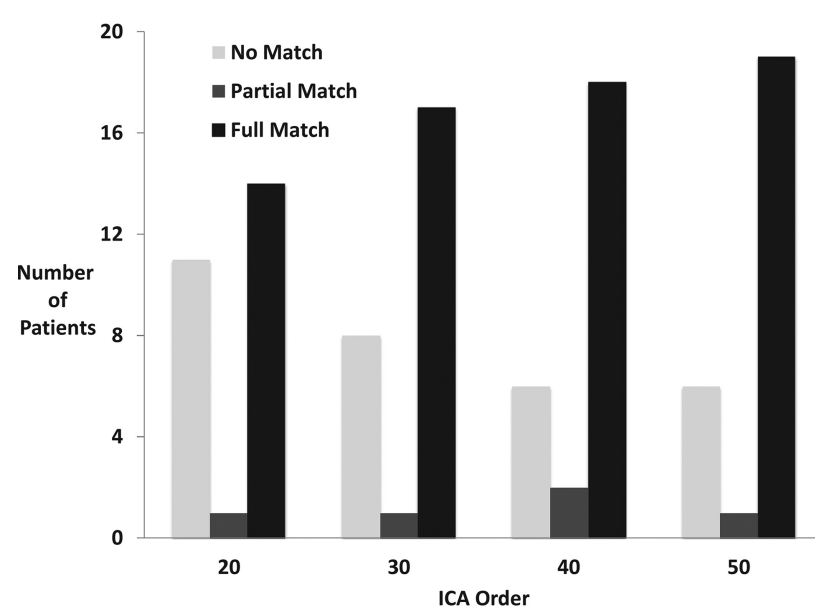

FIG 4. fMRI comparison among the patients. The number of patients with full, partial, and no match between tb- and rs-fMRI at different ICA levels is demonstrated. Table 1: Probability of getting a full match between rs-fMRI and
tb-fMRI maps as a function of number of ICA components

\begin{tabular}{ccccc}
\hline & ICA 20 & ICA 30 & ICA 40 & ICA 50 \\
\hline Probability & 0.53 & 0.65 & 0.69 & 0.73 \\
\hline
\end{tabular}

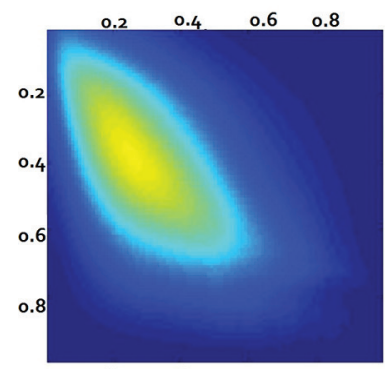

ICA 20

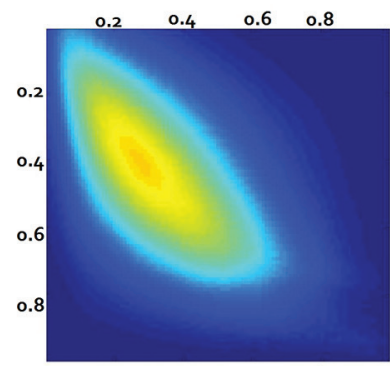

ICA 30

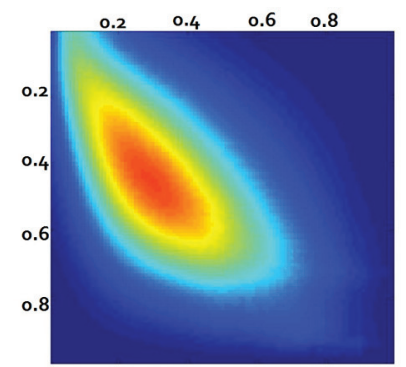

ICA 40

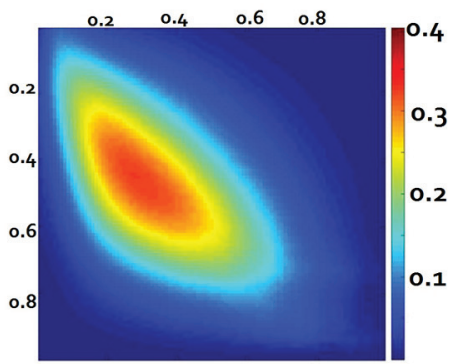

ICA 50

FIG 5. Group mean Dice coefficient maps across all the subjects at each ICA order calculated from subject-level noise-removed Dice coefficient maps. The $x$-axis depicts the rs-fMRI threshold levels, and the $y$-axis depicts the tb-fMRI threshold levels with the color bar demonstrating the Dice coefficient value. 


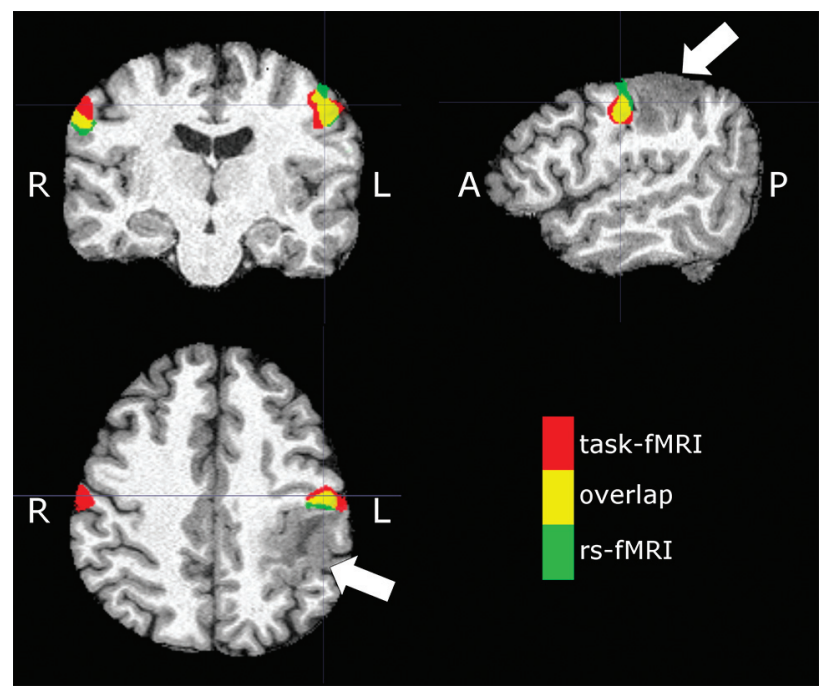

FIG 7. Sample subject (patient 23 in the On-line Table) demonstrating an expansile mass lesion centered in the postcentral gyrus (arrows). Signal abnormality is extended to the subcortical white matter of the precentral gyrus. Red denotes tongue motor task activation, green denotes the VSMN network identified from rs-fMRI (ICA 50), and yellow denotes areas of overlap between tb- and rs-fMRI.

Our data demonstrate the ability of rs-fMRI to successfully identify the VSMN in most patients (81\%). There was a significant increase in the number of rs- versus tb-fMRI full matches, with an increase in the number of ICA orders from 20 to 50. In addition, there was an increase in the mean Dice VUS and maximum Dice coefficient values, with an increasing number of independent components; however, this effect did not reach statistical significance, potentially due to intersubject variability. The choice of an ideal number of informative components for ICA analysis is challenging due to the spatial and temporal dependence of the BOLD signal. ${ }^{26}$ Using a higher number of target components could result in fragmentation of networks to subnetworks; conversely, using a lower number of target components may result in merging different brain networks. ${ }^{7}$ Concordantly, we qualitatively saw better separation of the VSMN and dorsal somatomotor network subnetworks at higher ICA orders. Therefore, using a higher number of target components in ICA analysis may be suggested in cases in which localizing a specific subnetwork is desired for preoperative planning, though this needs to be further balanced by the risk of further subsegmenting the networks with even higher ICA orders.

Our results show a strong concordance between the rs-fMRI and tb-fMRI in some of the subjects, demonstrating the potential utility of rs-fMRI as a viable preoperative mapping tool (Fig 7); however, there is significant variability across subjects. In particular, if rs-fMRI is to be used without a tb-fMRI acquisition, one must ensure that a reliability estimate of rs-fMRI can be calculated from the data itself. Improvement in technique and data analysis may overcome some of these limitations in the future. Increasing the scan time may improve the quality of rs- $-\mathrm{MRI}^{27}$ and thus potentially decrease such intersubject variability. Alternative methods of analysis such as seed-based analysis that have shown some promise in localizing the motor network in patients with brain tumors ${ }^{11,28}$ may also be considered. However, in the current study population of patients with brain tumors, accurate placement of seeds may be limited due to anatomic gyral distortion or compression. Therefore, an unbiased method such as ICA may be preferable. In addition, ICA remains an attractive choice due to availability of easy-to-use software such as the MELODIC tool in FSL (http://fsl.fmrib.ox.ac.uk/fsl/fslwiki/MELODIC) or the GIFT toolbox.

Several limitations in this study should be addressed. rs-fMRI was performed after tb-fMRI in this clinical cohort of patients to ensure that the patients tolerated the lengthy scan and performed well on their tb-fMRI, which was critical for clinical presurgical mapping. Thus, there may have been an inadvertent task effect on the observed functional connectivity. ${ }^{29}$ Another limitation is the inability to ensure that patients did not fall asleep during rs-fMRI. We instructed the patients to stay awake during the rs-fMRI and confirmed that they stayed awake during a postscan interview; however, the accuracy of their statements could not be verified by physiologic measures indicating sleep during the acquisition. While this inability poses a potential limitation because changes in functional connectivity have been reported in sleep or altered consciousness, ${ }^{30}$ functional connectivity in the somatomotor network has been shown to be preserved during different states of arousal. ${ }^{8}$ Nevertheless, the effect of sleep or altered arousal on rs-fMRI concordance with tb-fMRI could be further investigated. In addition, we used tb-fMRI T-maps to find the best candidate ICA map to represent the VSMN. However, brain tumors may cause alteration of somatomotor network organization, and brain tumor-induced neurovascular uncoupling may further compromise our ability to accurately detect the VSMN. Therefore, using only rs-fMRI data to identify the best somatomotor map may be challenging with brain tumors. The use of a data-driven neural network algorithm to identify the eloquent cortex in 7 patients with brain tumor with distorted anatomy has shown some promise. ${ }^{25}$

\section{CONCLUSIONS}

We demonstrate variable concordance of rs- and tb-fMRI at the single-subject level for detection of the VSMN in patients with brain tumor. We demonstrate improved reliability of rs-fMRI VSMN maps with higher ICA orders. Failure of rs-fMRI to identify the VSMN in about one-fifth of patients in our study limits the ability of rs-fMRI to completely substitute for tb-fMRI for presurgical assessment, though it may be considered as a supplement for tb-fMRI, for example, when task activation is suboptimal. Further improvements in technique, processing, and analysis methods may enhance the potential of using rs-fMRI as a substitute or complement to tb-fMRI in mapping of the VSMN.

Disclosures: Jay J. Pillai-UNRELATED: Royalties: Springer Science and Business Media, Elsevier, Comments: royalties for books published; Board Membership: Prism Clinical Imaging, Comments: unpaid medical advisory board member. Raag AiranUNRELATED: Grants/Grants Pending: Philips Healthcare, Comments: limited grant research support*; Stock/Stock Options: Lumos labs, Comments: stock options received as member of the Scientific Advisory Board. Brian Caffo-UNRELATED: Grant: National Institutes of Health, Comments: standard National Institutes of Health support.* Haris I. Sair-RELATED: Grant: Radiological Society of North America-Carestream Health Research Scholar Grant*; UNRELATED: Grants/Grants Pending: ImmunArray, Comments: grant support for research. * ${ }^{*}$ Money paid to the institution.

AJNR Am J Neuroradiol 38:1006-12 May 2017 www.ajnr.org 1011 


\section{REFERENCES}

1. Lehéricy S, Duffau H, Cornu P, et al. Correspondence between functional magnetic resonance imaging somatotopy and individual brain anatomy of the central region: comparison with intraoperative stimulation in patients with brain tumors. J Neurosurg 2000;92: 589-98 CrossRef Medline

2. Petrella JR, Shah LM, Harris KM, et al. Preoperative functional MR imaging localization of language and motor areas: effect on therapeutic decision making in patients with potentially resectable brain tumors. Radiology 2006;240:793-802 CrossRef Medline

3. Vlieger EJ, Majoie CB, Leenstra S, et al. Functional magnetic resonance imaging for neurosurgical planning in neurooncology. Eur Radiol 2004;14:1143-53 CrossRef Medline

4. Ogawa S, Menon RS, Tank DW, et al. Functional brain mapping by blood oxygenation level-dependent contrast magnetic resonance imaging: a comparison of signal characteristics with a biophysical model. Biophys J 1993;64:803-12 CrossRef Medline

5. Shimony JS, Zhang D, Johnston JM, et al. Resting-state spontaneous fluctuations in brain activity: a new paradigm for presurgical planning using fMRI. Acad Radiol 2009;16:578-83 CrossRef Medline

6. Sair HI, Yahyavi-Firouz-Abadi N, Calhoun VD, et al. Presurgical brain mapping of the language network in patients with brain tumors using resting-state fMRI: comparison with task fMRI. Hum Brain Mapp 2016;37:913-23 CrossRef Medline

7. Smith SM, Fox PT, Miller KL, et al. Correspondence of the brain's functional architecture during activation and rest. Proc Natl Acad Sci U S A 2009;106:13040 - 45 CrossRef Medline

8. Larson-Prior LJ, Zempel JM, Nolan TS, et al. Cortical network functional connectivity in the descent to sleep. Proc Natl Acad Sci U S A 2009;106:4489-94 CrossRef Medline

9. Mhuircheartaigh RN, Rosenorn-Lanng D, Wise R, et al. Cortical and subcortical connectivity changes during decreasing levels of consciousness in humans: a functional magnetic resonance imaging study using propofol. J Neurosci 2010;30:9095-102 CrossRef Medline

10. Zhang D, Johnston JM, Fox MD, et al. Preoperative sensorimotor mapping in brain tumor patients using spontaneous fluctuations in neuronal activity imaged with functional magnetic resonance imaging: initial experience. Neurosurgery 2009;65(6 suppl):226-36 Medline

11. Rosazza C, Aquino D, D'Incerti L, et al. Preoperative mapping of the sensorimotor cortex: comparative assessment of task-based and resting-state FMRI. PLoS One 2014;9:e98860 CrossRef Medline

12. Kokkonen SM, Nikkinen J, Remes J, et al. Preoperative localization of the sensorimotor area using independent component analysis of resting-state fMRI. Magn Reson Imaging 2009;27:733-40 CrossRef Medline

13. Tie Y, Rigolo L, Norton IH, et al. Defining language networks from resting-state fMRI for surgical planning: a feasibility study. Hum Brain Mapp 2014;35:1018-30 CrossRef Medline

14. Kristo G, Rutten GJ, Raemaekers M, et al. Task and task-free FMRI reproducibility comparison for motor network identification. Hum Brain Mapp 2014;35:340-52 CrossRef Medline
15. Hou BL, Bhatia S, Carpenter JS. Quantitative comparisons on hand motor functional areas determined by resting state and task BOLD fMRI and anatomical MRI for pre-surgical planning of patients with brain tumors. Neuroimage Clin 2016;11:378-87 CrossRef Medline

16. Schneider FC, Pailler M, Faillenot I, et al. Presurgical assessment of the sensorimotor cortex using resting-state fMRI. AJNR Am J Neuroradiol 2016;37:101-07 CrossRef Medline

17. Zacà D, Jovicich J, Nadar SR, et al. Cerebrovascular reactivity mapping in patients with low grade gliomas undergoing presurgical sensorimotor mapping with BOLD fMRI. J Magn Reson Imaging 2014;40:383-90 CrossRef Medline

18. Voyvodic JT. Reproducibility of single-subject fMRI language mapping with AMPLE normalization. J Magn Reson Imaging 2012;36: 569-80 CrossRef Medline

19. Mazaika PK. Methods and software for fMRI analysis of clinical subjects. Neuroimage 2009;47:S58 CrossRef

20. Behzadi Y, Restom K, Liau J, et al. A component based noise correction method (CompCor) for BOLD and perfusion based fMRI. Neuroimage 2007;37:90-101 CrossRef Medline

21. Himberg J, Hyvärinen A, Esposito F. Validating the independent components of neuroimaging time series via clustering and visualization. Neuroimage 2004;22:1214-22 CrossRef Medline

22. Branco P, Seixas D, Deprez S, et al. Resting-state functional magnetic resonance imaging for language preoperative planning. Front Hum Neurosci 2016;10:11 CrossRef Medline

23. Qiu $\mathrm{T}$, Yan $\mathrm{C}$, Tang W, et al. Localizing hand motor area using resting-state fMRI: validated with direct cortical stimulation. Acta Neurochir (Wien) 2014;156:2295-302 CrossRef Medline

24. Liu H. Task-free presurgical mapping using functional magnetic resonance imaging intrinsic activity. J Neurosurg 2009;111:746-54 CrossRef Medline

25. Mitchell TJ, Hacker CD, Breshears JD, et al. A novel data-driven approach to preoperative mapping of functional cortex using resting-state functional magnetic resonance imaging. Neurosurgery 2013;73:969-82; discussion 982-83 CrossRef Medline

26. Li YO, Adali T, Calhoun VD. Estimating the number of independent components for functional magnetic resonance imaging data. Hum Brain Mapp 2007;28:1251-66 CrossRef Medline

27. Birn RM, Molloy EK, Patriat R, et al. The effect of scan length on the reliability of resting-state fMRI connectivity estimates. Neuroimage 2013;83:550-58 CrossRef Medline

28. Agarwal S, Sair HI, Yahyavi-Firouz-Abadi N, et al. Neurovascular uncoupling in resting state fMRI demonstrated in patients with primary brain gliomas. J Magn Reson Imaging 2016;43:620-26 CrossRef Medline

29. Wang Z, Liu J, Zhong N, et al. Changes in the brain intrinsic organization in both on-task state and post-task resting state. Neuroimage 2012;62:394-407 CrossRef Medline

30. Tagliazucchi E, Laufs H. Decoding wakefulness levels from typical fMRI resting-state data reveals reliable drifts between wakefulness and sleep. Neuron 2014;82:695-708 CrossRef Medline 